\title{
Resilience Enhancement with Sequentially Proactive Operation Strategies
}

\author{
Chong Wang, Student Member, IEEE, Yunhe Hou, Senior Member, IEEE, Feng Qiu, Member, IEEE, \\ Shunbo Lei, Student Member, IEEE, and Kai Liu
}

\begin{abstract}
Extreme weather events, many of which are climate change-related, are occurring with increasing frequency and intensity and causing catastrophic outages, reminding the need to enhance the resilience of power systems. This paper proposes a proactive operation strategy to enhance system resilience during an unfolding extreme event. The uncertain sequential transition of system states driven by the evolution of extreme events is modeled as a Markov process. At each decision epoch, the system topology is used to construct a Markov state. Transition probabilities are evaluated according to failure rates caused by extreme events. For each state, a recursive value function, including a current cost and a future cost, is established with operation constraints and inter-temporal constraints. An optimal strategy is established by optimizing the recursive model, which is transformed into a mixed integer linear programming by using the linear scalarization method, with the probability of each state as the weight of each objective. The IEEE 30-bus system, the IEEE 118-bus system, and a realistic provincial power grid are used to validate the proposed method. The results demonstrate that the proposed proactive operation strategies can reduce the loss of load due to the development of extreme events.
\end{abstract}

Index Terms-Extreme weather events, generation redispatch, Markov model, power system resilience, sequentially proactive strategy

\section{NOMENCLATURE}

Indices and Sets

$i, i^{\prime}, i^{\prime \prime} \quad$ Index of Markov states

$t, t^{\prime} \quad$ Index of time periods

$a, a^{\prime} \quad$ Index of actions

$l \quad$ Index of lines

$k \quad$ Index of electrical devices

This work was supported by the National Natural Science Foundation of China under Grant 51677160, the Theme-based Research Scheme through Project No. T23-701/14-N, the Research Grant Council of Hong Kong SRA under Grants ECS739713 and GRF17202714, Basic Research Program-Shenzhen Fund (JCYJ20150629151046877), and research funding from China Southern Power Grid (ZD2014-2-0004).

C. Wang, Y. Hou, and S. Lei are with the Department of Electrical \& Electronic Engineering, The University of Hong Kong, Pokfulam, Hong Kong, and HKU Shenzhen Institute of Research and Innovation, Shenzhen, China (wangc@eee.hku.hk, yhhou@eee.hku.hk, leishunbo@eee.hku.hk).

F. Qiu is with the Energy Systems Division, Argonne National Laboratory, Argonne, IL 60439 USA (e-mail: fqiu@anl.gov ).

K. Liu is with China Southern Power Grid Co., Ltd., Guangzhou, China (liukai@.csg.cn) j Index of generators

$n, n^{\prime} \quad$ Index of nodes

$r \quad$ Index of paths between states

$\Omega_{C, t} \quad$ Set of components that might be in failure at $t$

$\Omega_{S, t} \quad$ Set of Markov states at $t$

$\Omega_{A} \quad$ Set of actions

$\Omega_{T} \quad$ Set of time periods

$\Omega_{N} \quad$ Set of nodes

$\Omega_{G} \quad$ Set of generators

$\Omega_{i, t+1}^{S} \quad$ Set of states at $t+1$ following the state $S_{i, t}$ at $t$

$\Omega_{i, t+}^{S} \quad$ Set of states

$\Omega_{n}^{G} \quad$ Set of generators connected with node $n$

$\Omega_{n}^{D} \quad$ Set of loads connected with node $n$

$\Omega_{n}^{N} \quad$ Set of nodes connected with node $n$

$\Omega_{i}^{\text {Path }} \quad$ Set of paths from initial state to state $S_{i, t}$

Notation for Failure Rate

$\lambda_{t}^{w} \quad$ Failure rate due to typhoon, windstorms etc

$w \quad$ A given wind speed

$w_{t} \quad$ Wind speed at $t$

$\alpha \quad$ A given parameter

$\lambda \quad$ Failure rate under normal weather conditions

$\lambda_{t}^{I} \quad$ Failure rate due to ice storm

$M_{t, L} \quad$ Total load in $\mathrm{kN}$ on line

$\bar{M}_{L} \quad$ Maximum load in $\mathrm{kN}$ on line

$m_{L i} \quad$ Ice load on lines

$m_{L w} \quad$ Wind load on lines

$f_{L}(\cdot) \quad$ Joint probability density function of ice load and wind load on lines

Notation for Optimization Model

$S_{i, t} \quad$ Markov state at $t$

$S_{i^{\prime}, t+1} \quad$ Markov state at $t+1$

$S_{i^{\prime \prime}, t^{\prime}} \quad$ Markov state at $t^{\prime}$

$s_{k, t} \quad$ Status of component $k$ at $t$ and $t+1$. ' 0 ' and ' 1 ' denote a failure state and normal operating status

$\lambda_{k, t+1} \quad$ Failure rate of component $k$ at $t+1$

$R_{t}(\cdot) \quad$ Immediate cost at $t(\$)$ 
$\operatorname{Pr}(\cdot) \quad$ Transition probability

$\gamma_{L, t} \quad$ Penalty due to loss of load (\$/MWh)

$v_{t}(\cdot) \quad$ Value function at $t$

$v_{t+1}(\cdot) \quad$ Value function at $t+1$

$v_{t}^{*}(\cdot) \quad$ Optimal value function

$L_{n, t, i} \quad$ Load demand of node $n$ in state $S_{i, t}$ at $t$ (MW)

$P_{j}^{\min } \quad$ Lower generation of generator $j$ (MW)

$P_{j}^{\max } \quad$ Upper generation of generator $j(\mathrm{MW})$

$R_{j}^{U P} \quad$ Ramp-up rate limit of generator $j(\mathrm{MW} / \mathrm{h})$

$R_{j}^{D N} \quad$ Ramp-down rate limit of generator $j(\mathrm{MW} / \mathrm{h})$

$P_{n, n^{\prime}}^{L, \max } \quad$ Max capacity of line $n-n^{\prime}(\mathrm{MW})$

$P_{n, n^{\prime}}^{L, \min } \quad$ Min capacity of line $n-n^{\prime}(\mathrm{MW})$

$\theta_{n}^{\max } \quad$ Max limit of Phase angle of node $n$

$\theta_{n}^{\min } \quad$ Min limit of Phase angle of node $n$

$A_{a, t} \quad$ Action $a$ at $t$

$B_{n, n^{\prime}} \quad$ Electrical susceptance of line $n-n^{\prime}$

$D_{j}^{O N} \quad$ Min on time of generator $j$ (Decision Epoch)

$D_{j}^{\text {OFF }} \quad$ Min off time of generator $j$ (Decision Epoch)

$\Delta T \quad$ Duration of each period (h)

$P_{j, t, i}^{G} \quad$ Generation of generator $j$ in state $S_{i, t}$ at $t(\mathrm{MW})$

$P_{j, t+1, i^{\prime}}^{G} \quad$ Generation of generator $j$ in state $S_{i^{\prime}, t+1}$ at $t(\mathrm{MW})$

$P_{n, n^{\prime}, t, i}^{L} \quad$ Power from node $n$ to $n^{\prime}$ in state $S_{i, t}$ at $t$ (MW)

$\Delta L_{n, t, i} \quad$ Load shedding of node $n$ in state $S_{i, t}$ at $t$ (MW)

$\theta_{n, t, i} \quad$ Phase angle of node $n$ in state $S_{i, t}$ at $t$

$o_{j, t, i} \quad$ Binary variable to indicate status of generator $j$ in state $S_{i, t}$ at $t$

$o_{j, t+1, i^{\prime}} \quad$ Binary variable to indicate status of generator $j$ in state $S_{i^{\prime}, t+1}$ at $t+1$

$o_{j, t^{\prime}, i^{\prime \prime}} \quad$ Binary variable to indicate status of generator $j$ in state $S_{i^{\prime \prime}, t^{\prime}}$ at $t^{\prime}$

$u_{n, n^{\prime}, t, i} \quad$ Binary outage indicator to indicate status of line $n-n^{\prime}$ in state $S_{i^{\prime \prime}, t^{\prime}}$ at $t$

$N_{T} \quad$ Number of time periods

$N \quad$ A large number

$P_{i}^{(P D F)} \quad$ Probability of state $S_{i, t}$

$p_{r, i, t} \quad$ Probability from initial state to state $S_{i, t}$ via path $r$

\section{INTRODUCTION}

$\mathrm{E}$ XTREME weather events, e.g., wind storms, typhoons, and hurricanes, are occurring with increasing intensity [1][2] and causing complete or partial power outages. These outages suggest the vulnerability of current power systems. Since power systems are critical infrastructures for society and economic development [3], an outage might cause severe consequences. In the United States, weather-related outages cause estimated $\$ 25$ billion economic losses each year [4]. In China, Typhoon Rammasun, which struck the Guangdong province on July 2014, took several $220 \mathrm{kV}$ transmission lines out of service. The severe consequences of such extreme weather events in power systems have brought power system resilience to the attention of organizations and governments in the world. A Policy Framework for the 21 ${ }^{\text {st }}$ Century Grid [5], which was released by the U.S. government in June 2011, emphasized the significance of resilient grids in countering the effects of increasingly intense weather events. The United States National Research Council (NRC) [3] and the House of Lords in the United Kingdom [6] have also emphasized the importance of a resilient energy infrastructure. The North American Electric Reliability Corporation (NERC) [7]-[9] and the United States Electric Power Research Institute (EPRI) [10] have both recognized the functionalities of system resilience.

Based on the requirements of power system resilience [3], some conceptual frameworks have been proposed [11]. To ensure resilience against extreme weather events, the strategies in the three stages of a severe event [8] (i.e., prior to the event, during the event and after the event) should be considered.

Prior to an extreme weather-related event, an accurate outage prediction contributes to manage preparedness and restoration efforts. To improve accuracy of predictions, a negative binomial regression model is proposed [12]. Since this model is based on data regarding outages caused by three hurricanes, i.e., Fran (1996), Bonnie (1998), and Floyd (1999), it is only suitable for a specific service area. To overcome this limit, a generic model for the full U.S. coastline is proposed in [13]. To estimate power outage durations in face of hurricanes, a statistical model is proposed in [14]. With outages and the duration predictions, some preventive strategies prior to an extreme weather-related event can be performed to increase power system resilience. Considering the stochastic and sequential characteristics of events, the events' potential impacts on the resilience of power systems are analyzed by using sequential Monte Carlo simulations [15]. To minimize negative impacts, the response before a hurricane is modeled as a mixed-integer programming problem [16]. In addition, preparation of sufficient blackstart generating units and emergency generators also plays an important role in improving power system resilience before an extreme weather-related event. To assess blackstart capacities, a GRM-based algorithm is developed [17]. To provide enough blackstart resources at right locations, [18] proposes a model to establish a procurement plan with a minimal cost while guarantee sufficient blackstart capacities. [19] focuses on dispatch strategies of mobile emergency generators to minimize the loss of load. Furthermore, some strategies, e.g., maintenance planning [20] and wide-area controls in response to communication failures [21], [22], can also be performed to enhance power system resilience before an weather-related event.

During the event, hardening, which refers to physically changing power systems, is a measure to make systems less susceptible to weather-related events. In [23], a resilient 
distribution network planning problem is formulated as a two-stage robust optimization model. In addition to hardening, islanding schemes can also be used to improve power system resilience. In [24], a unified resilience evaluation and an enhancement method, including a novel defensive islanding algorithm, are proposed. The proposed islanding scheme can mitigate potential cascading effects during weather-related events. Considering non-dispatchable and dispatchable distributed generators, [25] proposes a novel comprehensive operation and self-healing scheme, which sectionalizes a distribution system into several micro-grids, to improve distribution system resilience.

After an extreme weather-related event, it is necessary for system operators to implement restoration strategies [26]-[28] to restore loads as quickly as possible. A generic conventional power system restoration can be divided into three stages, i.e., preparation, system restoration and load restoration [29]. In the preparation stage, the system status, i.e., blackstart units, non-blackstart units and critical loads, should be evaluated. In the system restoration stage, the main goal is to establish a strong bulk power network by restarting appropriate blackstart and non-blackstart units associated with appropriate transmission lines and some critical loads [30]. In the load restoration stage, the critical objective is to restore all loads as quickly as possible. Many approaches, e.g., expert systems [31] and heuristic approaches [32], have been proposed to deal with load restoration. However, outages caused by weather-related events usually have their own unique characteristics, which might result in inapplicability of the existing recovery schemes. Therefore, new techniques should be proposed to deal with restoration after weather-related events. [28] proposes a novel operational approach for distribution systems by establishing multiple microgrids energized distributed generators to restore critical load from power outages. In [33], the impact of microgrids as blackstart resources after a natural disaster is evaluated. Furthermore, decentralized restoration schemes [34] can be employed.

Most research studies have focused on assessment/strategies prior to an weather-related event and restoration strategies after an weather-related event. However, strategies during an event are still in their infancy. In this work, we focus on operational strategies during an event to enhance power system resilience against extreme weather-related events. During extreme weather events, operating strategies should be established subject to both current system/equipment statuses and potential future statuses as the weather-related events unfolding. Due to the essentially sequential characteristics during an event unfolding, the operation strategies should be a sequence of actions associated with uncertainties caused by development of the event and faults of components.

The main contributions of this paper are two-fold. 1) A Markovian method for sequentially proactive generation redispatch is proposed. At each decision epoch, the system topology, which may change due to the failure of some components (such as transformers or transmission lines) due to extreme event, constitutes a Markov state. Transition probabilities between different states, i.e., different topologies, are determined by component failure rates and development of the event. In each state, a recursive value function that includes a current cost and a future cost is established subject to operation constraints (such as ramping rates of generators). 2) The optimal strategy for each state is obtained by optimizing the proposed recursive model. The recursive model is transformed into a mixed integer linear programming by using the linear scalarization method, with the probability of each state used as the weight of each objective. The linear programming is solved with the CPLEX solver. Two IEEE test systems and a modified realistic system are used to validate the proposed model, with the results showing that the proposed model provides insight for proactive generation redispatch under extreme weather events.

This paper is organized as follows. Section II describes the impacts of extreme weather events on system states. Section III introduces sequentially proactive operation strategies, while Section IV shows the solution. Section V presents the case studies, and the work is concluded in Section VI.

\section{INFLUENCES OF EXTREME WEATHER EVENTS ON SYSTEM STATES}

This section introduces the influences of extreme weather events on system states. First, several component failure rate models are introduced. Second, system states on the trajectory of extreme weather events are presented. Third, transition probabilities between different system states are modeled.

\section{A. Component Failure Rate}

In certain extreme weather events, such as hurricanes, tornados, typhoons, windstorms, floods, lightning storms [35], the intensity of the hazardous forces will change both temporally and geographically as the trajectories of the weather events move passing a region. The component failures are correlated with hazardous forces. Usually, a generic fragility curve, in Fig. 1, can be used to relate failure probabilities of a component to the weather intensity [24].

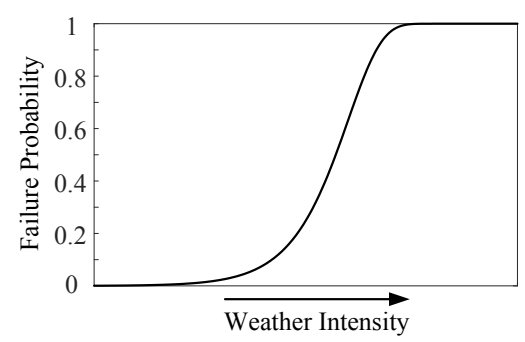

Fig. 1 Generic fragility curve

Typically, several models for calculating component failure probabilities under different extreme weather events may be employed.

1) Failure rate caused by hurricanes, tornados, typhoon and windstorms

For hurricanes, tornados, typhoons and windstorms, the pressure of high wind is a key reason of component failures. It is deemed that the pressure exerted on components is approximately proportional to the square of wind speeds [36]. 
The failure rates of components during these events can be expressed as

$$
\lambda_{t}^{w}=\left[1+\alpha \cdot\left(w_{t}^{2} / w^{2}-1\right)\right] \cdot \lambda
$$

\section{2) Failure rate caused by ice storm}

Many research studies have focused on transmission reliability assessment in consideration of ice storms. Take transmission lines for example, their failure rates under ice storms can be expressed as

$$
\lambda_{t}^{L}=\iint_{\bar{M}_{L}}^{M_{t, L}} f_{L}\left(m_{L i}, m_{L w}\right) d m_{L i} d m_{L w}
$$

The failure rates of transmission towers under ice storms have the similar expression.

\section{B. System States on Trajectories of Extreme Weather Events}

Because of the time sequence in which components experience extreme weather events, different components, e.g., lines, transformers, loads and generators, might be in failure at sequential time intervals. For example, there are two components A and B on the trajectory of a typhoon, as shown in Fig. 2. Since the failure rates depend on the wind speed, the failure rates of each component at different sequential time intervals are usually different, as shown in Fig. 3(a).

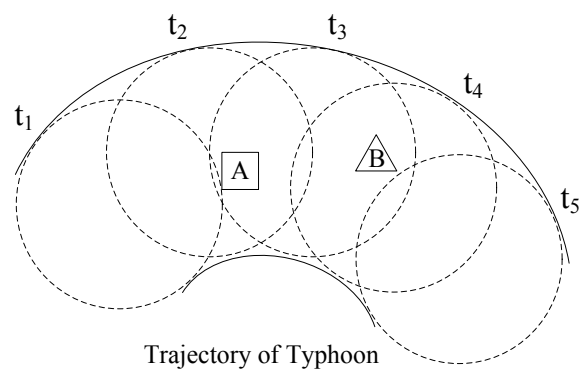

Fig. 2 Two components on the trajectory of a typhoon.

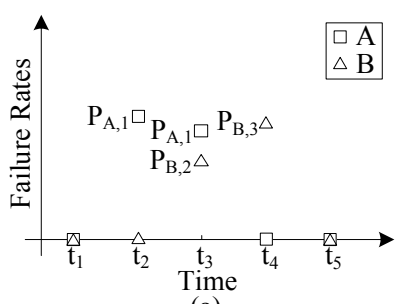

(a)

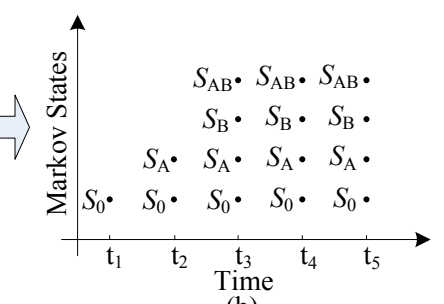

(b)
Fig. 3 (a) Failure rates at different time intervals. (b) Markov states at different time intervals.

At each decision epoch, the system topology may be changed due to component failures in the face of an event. As for the system operators, they should make decisions based on the current system topology at each decision epoch in the face of the event, as well as consider possible topology scenarios caused by the extreme weather event in the subsequent time intervals. In this paper, the system topology is defined as a Markov state. The number of Markov states depends on the number of components that might be in failure due to the extreme weather event. Let $\Omega_{\mathrm{C}, \mathrm{t}}$ be the set of components that might be in failure at time $t$. Sets at different time intervals satisfy the following equations.

$$
\Omega_{C, t} \subset \Omega_{C, t+1}, \quad t=\{1,2, \cdots\}
$$

where (3) denotes that possible failed components at one interval should include the possibly failed components from the previous time intervals. For example, the typhoon only directly influences the component B at $t_{3}$, but $\Omega_{C, \mathrm{t}}$ at $t_{3}$ in Fig. 2 includes the component $\mathrm{A}$ as well as the component B. The Markov states include $S_{0}, S_{A}, S_{B}$ and $S_{A B}$, as shown in Fig. 3(b). The subscript ' 0 ' denotes that no components are in failure. In general, the number of Markov states at time $t$ is $2^{N_{C, t}}$, and $N_{C, t}$ is the number of components that might be in failure at time $t$.

Usually, a realistic trajectory is uncertain and the forecast of the trajectory cannot be entirely accurate. When considering the uncertainty of a trajectory, its influences on component failures can be included in component failure probabilities.

\section{Transition Probabilities between Different System States}

Let $\Omega_{S, t}$ be the set of states at time $t$. Let $S_{i, t}$ and $S_{i^{\prime}, t+1}$ be states at time $t$ and $t+1$, respectively. The transition probability from $S_{i, t}$ to $S_{i^{\prime}, t}$ can be expressed as

$$
\begin{aligned}
& \operatorname{Pr}\left(S_{i, t}, S_{i^{\prime}, t+1}\right)=\prod_{k \in \Omega_{C, t+1}} \operatorname{Pr}\left(s_{k, t}, s_{k, t+1}\right), i \in \Omega_{S, t} \\
& \operatorname{Pr}\left(s_{k, t}, s_{k, t+1}\right)= \begin{cases}1 & s_{k, t}=0, s_{k, t+1}=0 \\
0 & s_{k, t}=0, s_{k, t+1}=1 \\
1-\lambda_{k, t+1} & s_{k, t}=1, s_{k, t+1}=1 \\
\lambda_{k, t+1} & s_{k, t}=1, s_{k, t+1}=0\end{cases}
\end{aligned}
$$

where $\operatorname{Pr}(\cdot)$ is the transition probability, $\lambda_{k, t+1}$ is the failure probability of the component $k$ at time $t+1, s_{k, t}$ and $s_{k, t+1}$ are statuses of the component $k$ at time $t$ and $t+1$, respectively. The lowercase $s$ denotes a status of an electrical device on the trajectory of a weather event. It can be a failure status or a non-failure status. ' 0 ' and ' 1 ' denote a failure status and a normal operating status, respectively. The uppercase $S$ denote a system state, which should consider all statuses of electrical devices on the trajectory of a weather event.

\section{Sequentially Proactive Operation Strategy}

This section introduces the optimization model to establish sequentially proactive operation strategies. First, a recursive model for each system state, including current and future influences, is established. Second, constraints for sequentially proactive operation strategies are presented.

\section{A. Sequential Decision Processes}

Since the failure of a component on the trajectory of an extreme weather event is uncertain, the best decision making for system operators is to adjust strategies, according to real-time states of the system, to optimize their objective. Meanwhile, when making decisions based on a state, the system operators should consider not only the current influences, but also any future influences caused by the decisions. This decision process is a Markov decision process. For a realistic system, the system operators make decisions continuously. To simplify the model, we assume that the decisions are made at discrete decision epochs. In this context, 
we model the whole decision process as a discrete-time Markov decision process.

Since the system operators should consider both current and future influences, the value function for each state can be expressed as a recursive formula.

$$
\begin{aligned}
v_{t}\left(S_{i, t}, A_{a, t}\right)=R_{t}\left(S_{i, t}, A_{a, t}\right)+ \\
\sum_{i^{\prime} \in \Omega_{i, t+1}^{S}}\left[\operatorname{Pr}\left(S_{i, t}, S_{i^{\prime}, t+1}\right) \cdot v_{t+1}\left(S_{i^{\prime}, t+1}, A_{a^{\prime}, t+1}\right)\right] \\
\quad i \in \Omega_{S, t}, a \in \Omega_{A}, a^{\prime} \in \Omega_{A}, t \in \Omega_{T}, t+1 \in \Omega_{T}
\end{aligned}
$$

where $A_{a, t}$ denotes actions, i.e., redispatching the system. In this paper, "redispatching the system" means "generation redispatch", i.e., adjusting outputs of available generators. $v_{t}\left(S_{i, t}, A_{a, t}\right)$ is the expected cost, from time $t$ to the terminal time, with state $S_{i, t}$ under action $A_{a, t}$. Before an event hits a power grid, the system topology can also be considered as a Markov state, which can be included in the model. $R\left(S_{i, t}, A_{a, t}\right)$ is the immediate cost at time $t$ for state $S_{i, t}$ under action $A_{a, t}$. This immediate cost is the cost of loss of load. Usually, when a system is under a severe weather event, the reliability, represented as expected loss of load in the paper, has a higher priority. Therefore, the expected loss of load works as the objective of the system operators.

Based on (6), the optimal strategy with state $S_{i, t}$ at $t$ can be obtained by using the following formula.

$$
v_{t}^{*}\left(S_{i, t}\right)=\min \left\{v_{t}\left(S_{i, t}, A_{a, t}\right), a \in \Omega_{A}\right\}, i \in \Omega_{S, t}, t \in \Omega_{T}
$$

where $v_{t}^{*}\left(S_{i, t}\right)$ is the minimal expected cost of state $S_{i, t}$ at time $t$. The immediate cost $R_{t}\left(S_{i, t}, A_{a, t}\right)$ is the cost of loss of load, which can be expressed as

$$
R_{t}\left(S_{i, t}, A_{a, t}\right)=\gamma_{L, t} \cdot \sum_{n \in \Omega_{N}}\left(\Delta L_{n, t, i} \cdot \Delta T\right)
$$

\section{B. Constraints for Sequentially Proactive Operation Strategy}

During generation redispatch in state $S_{i, t}$ at time $t$, the constraints of power balance, upper and lower limits of generators, upper and lower limits of voltage, power flows through lines and load limits should be satisfied. Typically, the ramping rates between possible states should be satisfied.

1) Power balance

The power balance constraint in state $S_{i, t}$ at time $t$ can be expressed as

$$
\sum_{j \in \Omega_{n}^{G}} P_{j, t, i}^{G}-\left(L_{n, t, i}-\Delta L_{n, t, i}\right)+\sum_{n^{\prime} \in \Omega_{n}^{N}} P_{n, n^{\prime}, t, i}^{L}=0, \forall n
$$

where (9) denotes power balance at each node in state $S_{i, t}$ at time $t$.

\section{2) Ramping rates of generators}

The ramping rates of each generator in state $S_{i, t}$ at time $t$ should be satisfied during the implementation of any proactive generation redispatch such that

$$
\begin{aligned}
P_{j, t+1, i^{\prime}}^{G}-P_{j, t, i}^{G} & \leq\left(2-o_{j, t, i}-o_{j, t+1, i^{\prime}}\right) \cdot P_{j}^{\min } \\
& +\left(1+o_{j, t, i}-o_{j, t+1, i^{\prime}}\right) \cdot R_{j}^{U P} \quad i^{\prime} \in \Omega_{i, t+1}^{S}, \forall j \\
P_{j, t, i}^{G}-P_{j, t+1, i^{\prime}}^{G} & \leq\left(2-o_{j, t, i}-o_{j, t+1, i^{\prime}}\right) \cdot P_{j}^{\min } \\
+ & \left(1-o_{j, t, i}+o_{j, t+1, i^{\prime}}\right) \cdot R_{j}^{D N} \quad i^{\prime} \in \Omega_{i, t+1}^{S}, \forall j
\end{aligned}
$$

where the term $i^{\prime} \in \Omega_{i, t+1}^{S}$ ensures that ramping rates should be satisfied between the state $S_{i, t}$ at $t$ and its possible following states at $t+1$. Considering potential online/offline statuses of generators, binary variables regarding generators' statuses are included in constraints (10) and (11).

3) Minimum up time and down time constraints of generators

When performing generation redispatch, minimum up time and down time constraints of generators should be satisfied.

$$
\begin{gathered}
-o_{j, t, i}+o_{j, t+1, i^{\prime}}-o_{j, t^{\prime}, i^{\prime \prime}} \leq 0,1 \leq t^{\prime}-t \leq D_{j}^{O N} \\
i^{\prime} \in \Omega_{i, t+1}^{S}, i^{\prime \prime} \in \Omega_{i, t+}^{S}, \forall j \\
o_{j, t, i}-o_{j, t+1, i^{\prime}}+o_{j, t^{\prime}, i^{\prime \prime}} \leq 1,1 \leq t^{\prime}-t \leq D_{j}^{O F F} \\
i^{\prime} \in \Omega_{i, t+1}^{S}, i^{\prime \prime} \in \Omega_{i, t+}^{S}, \forall j
\end{gathered}
$$

where the terms $i^{\prime} \in \Omega_{i, t+1}^{S}$ and $i^{\prime \prime} \in \Omega_{i, t+}^{S}$ ensure that minimum up time and down time constraints should be satisfied between possible transition states.

4) Power flows of lines

The limits for power flows through online lines in state $S_{i, t}$ at time $t$ should be satisfied.

$$
\begin{aligned}
& B_{n, n^{\prime}} \cdot\left(\theta_{n, t, i}-\theta_{n^{\prime}, t, i}\right)-P_{n, n^{\prime}, t, i}^{L}+\left(1-u_{n, n^{\prime}, t, i}\right) \cdot N \geq 0 \\
& n, n^{\prime} \in \text { Line }_{l}, \forall l \\
& B_{n, n^{\prime}} \cdot\left(\theta_{n, t, i}-\theta_{n^{\prime}, t, i}\right)-P_{n, n^{\prime}, t, i}^{L}-\left(1-u_{n, n^{\prime}, t, i}\right) \cdot N \leq 0 \\
& n, n^{\prime} \in \text { Line }_{l}, \forall l \\
& P_{n, n^{\prime}}^{L, \min } \cdot u_{n, n^{\prime}, t, i} \leq P_{n, n^{\prime}, t, i}^{L} \leq P_{n, n^{\prime}}^{L, \max } \cdot u_{n, n^{\prime}, t, i} \\
& n, n^{\prime} \in \text { Line }_{l}, \forall l
\end{aligned}
$$

where (14) and (15) represent the physical relations between voltage angles and power flows through transmission lines. $u_{n, n^{\prime}, t, i}$ is a binary outage indicator. If the line $l$ is in outage in state $S_{i, t}$ at time $t, u_{n, n^{\prime}, t, i}=0$; otherwise $u_{n, n^{\prime}, t, i}=1 . N$ is a disjunctive parameter. With a sufficiently large $N,(14)$ and (15) are redundant when lines are outages. (16) shows the limits of transmission lines. The models of power flows through transformers are similar to (14)-(16).

5) Upper and lower limits of the outputs of generators

During the implementation of generation redispatch in state $S_{i, t}$ at time $t$, the upper and lower limits of generators should be satisfied by

$$
P_{j}^{\min } \cdot o_{j, t, i} \leq P_{j, t, i}^{G} \leq P_{j}^{\max } \cdot o_{j, t, i} \quad \forall j
$$

6) Load limits

When performing generation redispatch in state $S_{i, t}$ at time $t$, load shedding might be conducted to ensure power balance when considering the ramping rates of the generators, power flows through the lines and so on. When conducting load shedding, the following constraints should be involved

$$
0 \leq \Delta L_{n, t, i} \leq L_{n, t, i} \quad \forall n
$$

where $L_{n, t, i}$ is the forecasted load of the bus $n$ at $t$. Its value can be predicted based on existing load forecasting methods.

\section{7) Voltage limits}

The following constraint regarding voltage in state $S_{i, t}$ at $t$ should be satisfied.

$$
\theta_{n}^{\min } \leq \theta_{n, t, i} \leq \theta_{n}^{\max } \quad \forall n
$$




\section{8) Other constraints}

For binary variables in state $S_{i, t}$ at $t$, there are following constraints.

$$
o_{j, t, i} \in\{0,1\} \quad \forall j
$$

where $o_{j, t, i}$ represents the generator's status. System operators can add constraints regarding binary variables to artificially set statuses of generators. In addition, some generators might be out of service, due to weather events, corresponding to certain Markov states. Under this condition, the constraint $o_{j, t, i}=0(j, t$ and $i$ corresponding to given values) are added.

\section{Solution Method}

According to (6) and (7), the optimal strategy for each state should ensure the minimum expected cost from the current decision epoch to the terminal decision epoch. Take the scenario in Fig. 4 as an example. For the state $S_{1}$, the optimal strategy should ensure the minimum expected cost of the current state and the future possible states, i.e., the states in the red triangle. For the state $S_{2}$, the optimal strategy should ensure the minimum expected cost of the states in the blue triangle. From the perspective of the mathematical model, it is necessary to find a solution for each state to guarantee that the multiple objectives are optimal. If the ramping rates of each generator are not considered, the backward induction method and the value iteration method [37] can be employed to find the solution for each state. However, if we include the ramping rates as a condition, it is difficult for some scenarios to find solutions for each state to ensure optimal multiple objectives simultaneously.

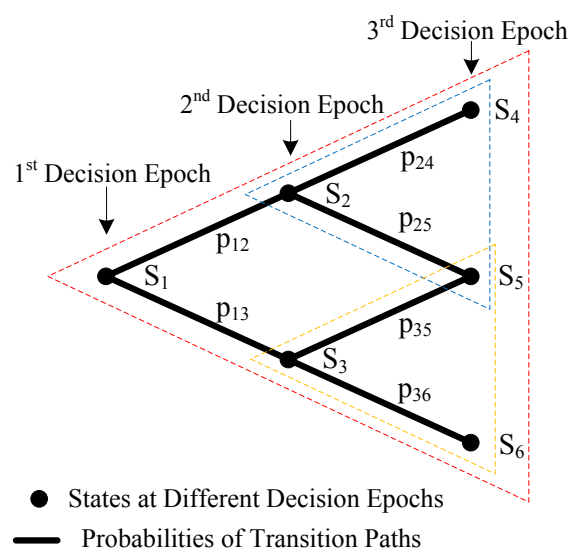

Fig. 4 A scenario with three decision epochs.

When considering the generators' ramping rates with (6) and (7), the problem becomes an optimization with multiple objectives. In this paper, we employ the linear scalarization method to transform the original multi-objective optimization problem into a single-objective optimization problem.

For the linear scalarization method, one critical point is to determine the weight of each objective. The probability of each state works as the weight of the objective of the corresponding state. Even though each state, i.e., a system topology, occurs stochastically due to the uncertain failure of each component on the trajectory of the extreme weather event, the probability of each state can be expressed as

$$
P_{i}^{(P D F)}=\frac{1}{N_{T}} \sum_{r \in \Omega_{i}^{\text {palh }}}\left(p_{r, i, t}\right)
$$

Take the scenario in Fig. 4 as an example, the probabilities of $S_{1}$, $S_{2}, S_{3}, S_{4}, S_{5}$ and $S_{6}$ are as shown in Fig. 5.

The linear scalarization method is used to reformulate the model as a mixed integer linear programming. The reformulated model is similar to a stochastic optimization. With the weight of each objective, the reformulated model can be expressed as follows.

$$
\min \sum_{t \in \Omega_{T}} \sum_{i \in \Omega_{S, t}}\left\{P_{i}^{(P D F)} \cdot v_{t}\left(S_{i, t}, A_{a, t}\right)\right\}
$$

subject to

Equations (4), (5), $i \in \Omega_{S, t}, t \in \Omega_{T}$

Equation (6)

Equation (8), $i \in \Omega_{S, t}, t \in \Omega_{T}$

Constraint (9), (10), (11),(12), (13), $i \in \Omega_{S, t}, t \in \Omega_{T}$

Constraints (14), (15), (17), (18),(19), (20), $i \in \Omega_{S, t}, t \in \Omega_{T}$

Equation (21), $i \in \Omega_{S, t}, t \in \Omega_{T}$.

The reformulated model is a mixed integer linear programming, which is solved using the CPLEX solver.

With increasing components on the trajectory of an extreme weather event and decision epochs, the state tree in Fig. 4 increase exponentially. The model may be computationally intractable. Scenario reduction, e.g., deleting states with extreme low probabilities, can be used to ensure acceptable computer velocity.

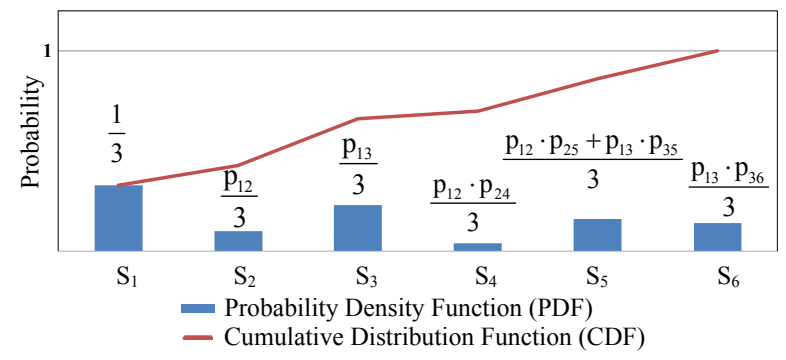

Fig. 5 Probabilities of states.

\section{CAse Studies}

In this section, we present simulations conducted using the IEEE 30-bus system, the IEEE 118-bus system and a practical power grid system to show the effectiveness of the proposed model. The cases are tested in MATLAB 2014b using the CPLEX solver on a personal computer with a $3.1 \mathrm{GHz}$ i5 processor and 8 GB RAM. For the sake of brevity, we take the extreme weather event of a typhoon as the event for each case.

\section{A. IEEE 30-bus System}

\section{1) Data description}

The IEEE 30-bus system and the trajectory of the typhoon are shown in Fig. 6. For the sake of exposition, we assume that decisions are made at discrete time $t_{1}, t_{2}, t_{3}, t_{4}$ and $t_{5}$. The duration between two adjacent decision epochs is 6 minutes. On the trajectory of the typhoon, the potential failure components include six transmission lines, two generators and two partial loads. Since distribution systems might be damaged, 
on this occasion, the load would decrease from the perspective of transmission systems. The failure rates of the components on the trajectory of the typhoon are shown in Table I. In practice, these probabilities can be achieved based on generic fragility curves or failure rate calculation models, e.g., (1).

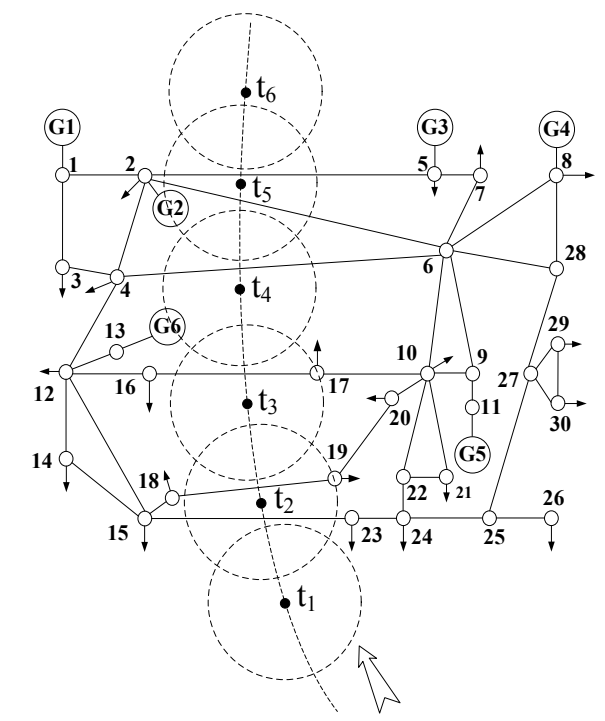

Fig. 6 IEEE 30-bus system topology.

TABLE I

PROBABILITIES OF COMPONENT FAILURE

\begin{tabular}{ccc}
\hline \hline Component No. & Components & Failure Probabilities \\
\hline$C_{1}$ & Line 15-23 & 0.25 \\
$C_{2}$ & Line 18-19 & 0.22 \\
$C_{3}$ & $50 \%$ Load 19 & 0.10 \\
$C_{4}$ & Line 16-17 & 0.20 \\
$C_{5}$ & $60 \%$ Load 17 & 0.10 \\
$C_{6}$ & $G_{6}$ & 0.08 \\
$C_{7}$ & Line 4-6 & 0.18 \\
$C_{8}$ & $G_{2}$ & 0.08 \\
$C_{9}$ & Line 2-6 & 0.15 \\
$C_{10}$ & Line 2-5 & 0.12 \\
\hline \hline
\end{tabular}

TABLE II

PARAMETERS OF GENERATORS

\begin{tabular}{ccccccc}
\hline \hline Unit & Bus & $P_{\min }$ & $P_{\max }$ & Ramping Rates & $D_{i}^{\text {ON }}$ & $D_{i}^{\text {OFF }}$ \\
\hline$G_{1}$ & 1 & 30 & 120 & 120 & 3 & 3 \\
$G_{2}$ & 2 & 35 & 140 & 120 & 3 & 3 \\
$G_{3}$ & 5 & 10 & 50 & 72 & 3 & 3 \\
$G_{4}$ & 8 & 05 & 30 & 60 & 3 & 3 \\
$G_{5}$ & 11 & 10 & 55 & 72 & 3 & 3 \\
$G_{6}$ & 13 & 15 & 40 & 60 & 3 & 3 \\
\hline \hline
\end{tabular}

\section{2) Mapping states to strategies}

Based on the proposed model, the optimal redispatch strategy for each state is calculated in advance. The strategies at the decision epoch $t_{1}$ can be considered as preventive actions before the typhoon. During the typhoon, the system operators observe the system topology, i.e., the state, at one decision epoch, and then map the state to the optimal strategy. The same process is repeated at the next decision epoch, as shown in Fig.
7. Table III shows four scenarios with uncertain failure components, and Fig. 8 shows the corresponding strategies. The optimal strategies are selected according to system states in real systems.

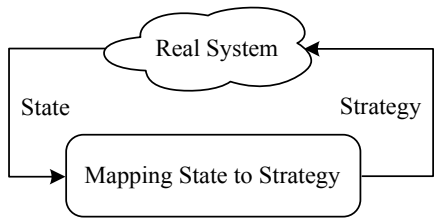

Fig. 7 The process of mapping a state to a strategy.

TABLE III

DIFFERENT FAILURE SCENARIOS

\begin{tabular}{cccccc}
\hline \hline $\begin{array}{c}\text { Scenario } \\
\text { No. }\end{array}$ & \multicolumn{5}{c}{ Failure Components } \\
\cline { 2 - 6 } & $t_{1}$ & $t_{2}$ & $t_{3}$ & $t_{4}$ & $t_{5}$ \\
\hline 1 & No & No & No & $C_{6}, C_{7}$ & $C_{6}, C_{7}, C_{9}, C_{10}$ \\
2 & No & $C_{3}$ & $C_{3}, C_{5}$ & $C_{3}, C_{5}, C_{6}, C_{7}$ & $C_{3}, C_{5}, C_{6}, C_{7}$, \\
& \multirow{2}{*}{ No } & $C_{1}$ & $C_{1}, C_{4}$ & $C_{1}, C_{4}, C_{7}$ & $C_{1}, C_{4}, C_{7}, C_{9}$ \\
3 & \multirow{2}{*}{$C_{2}, C_{3}$} & $C_{2}, C_{3}, C_{4}$, & $C_{2}, C_{3}, C_{4}, C_{5}$, & $C_{2}, C_{3}, C_{4}, C_{5}$, \\
4 & $C_{5}, C_{7}, C_{7}$ & $C_{6}, C_{7}, C_{9}, C_{10}$ \\
\hline \hline
\end{tabular}

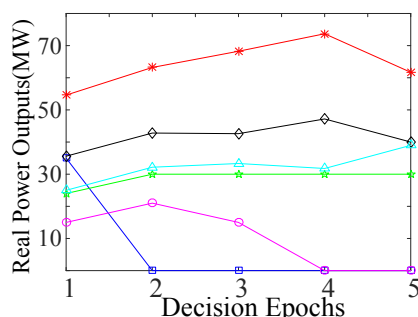

(a)

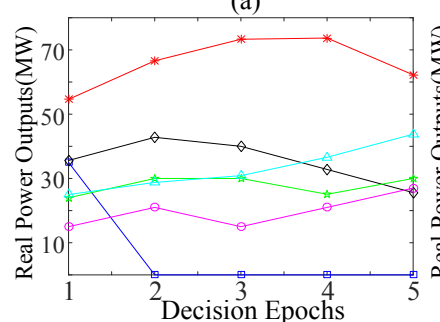

(c)

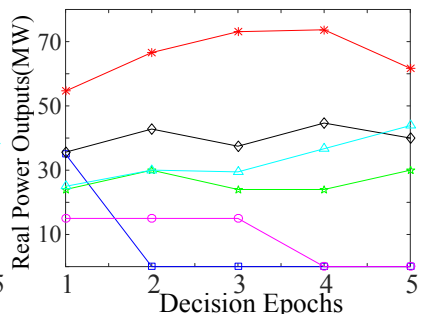

(b)

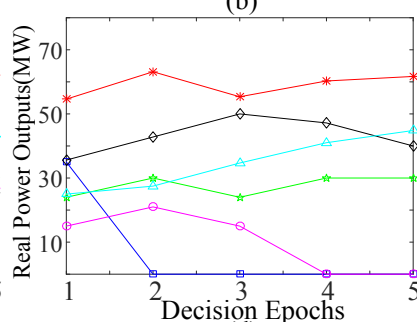

(d)

Fig. 8 Optimal strategies for four scenarios.

Since the generators $G_{2}$ and $G_{6}$ might be in failure on the typhoon's trajectory, these two generators are tended to be offline or with small outputs in the process of generation dispatch. For example, the generator $G_{6}$ has small outputs so that the other generators can cover its generation loss due to the typhoon, as shown in Fig. 8 (a), (b) and (d).

The strategies should also consider potential damaged load due to the typhoon. For example, the total generation at the first decision epoch is $189.2 \mathrm{MW}$. At the second decision epoch, the total generation becomes $184.45 \mathrm{MW}$ if $50 \%$ load at the bus 19 is damaged due to the typhoon in Fig. 8 (b), while the total generation retain 189.2 MW if the load is not damaged. Similarly, the total generation becomes $179.05 \mathrm{MW}$ if $60 \%$ load at the bus 17 is damaged at the third decision epoch, while retain $189.2 \mathrm{MW}$ if not.

Fig. 9 shows the strategies for possible states at each decision 
epoch. Based on these mapping relations, the system operators can select the optimal strategy for the observed state at each decision epoch.

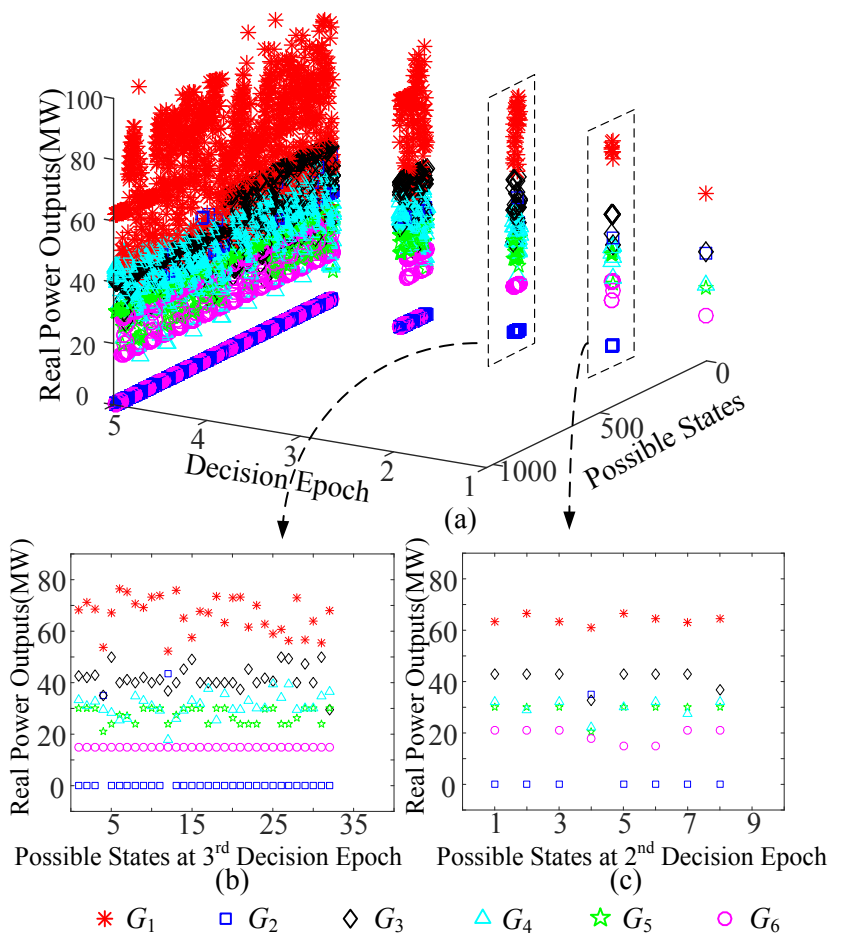

Fig. 9 Mapping states to strategies.

\section{3) Effectiveness of the proposed method}

This section shows the effectiveness of the proposed method by comparing the following methods:

M1: The proposed method;

M2: Non-proactive strategies. In this case, system operators will not proactively perform generation redispatch beforehand. They only take actions after some events, i.e., line faults, to minimize loss of load, in consideration of operation constraints.

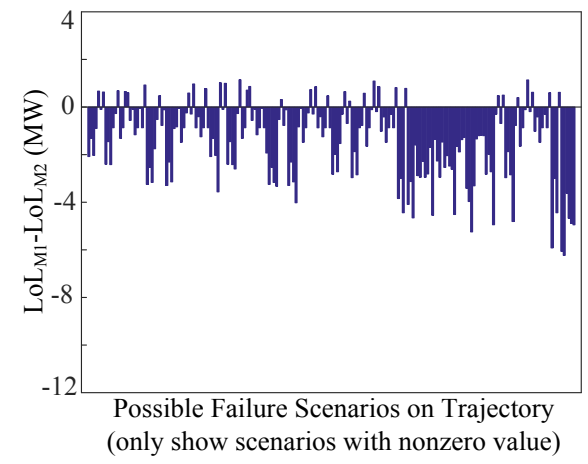

Fig. 10 Differences of LoL with M1 and M2.

Fig. 10 shows differences of loss of load (LoL) with M1 and M2. Most failure scenarios correspond to smaller loss of load by using M1. There are a few failure scenarios with larger loss of load by using M1, because M1 synthetically considers possible scenarios on the trajectory of the typhoon and some scenarios may sacrifice loss of load. From the perspective of all scenarios, however, the proposed method is more effective.

\section{4) Influences of scenario reduction}

This section shows the influences of state reduction. Table IV shows the results of different maximum failure component scenarios. More maximum failure components have higher accuracy, while result in longer CPU time.

TABLE IV

DIFFERENT MAXIMUM FAILURE COMPONENT SCENARIOS

\begin{tabular}{cccc}
\hline \hline $\begin{array}{c}\text { Maximum Failure } \\
\text { Components }\end{array}$ & $\begin{array}{c}\text { Objective } \\
\text { Value }(\$)\end{array}$ & $\begin{array}{c}\text { Error } \\
(\%)\end{array}$ & $\begin{array}{c}\text { Total CPU } \\
\text { Time (s) }\end{array}$ \\
\hline 2 & 322.54 & 47.92 & 29 \\
3 & 476.42 & 23.07 & 72 \\
4 & 569.55 & 8.03 & 185 \\
5 & 603.90 & 2.49 & 542 \\
6 & 616.06 & 0.52 & 1035 \\
7 & 618.94 & 0.06 & 1935 \\
8 & 619.27 & 0.0048 & 3021 \\
9 & 619.28 & 0.0032 & 3935 \\
10 & 619.30 & 0 & 4235 \\
\hline \hline
\end{tabular}

\section{B. IEEE 118-bus System}

\section{1) Data description}

The IEEE 118-bus system and the trajectory of a typhoon are shown in Fig. 11. The data for the system can be found in [38] and [39]. The ramping rates of all generators are assumed to be $60 \mathrm{MW} / \mathrm{h}$. For the sake of exposition, decisions are made at discrete times $t_{1}, t_{2}, t_{3}, t_{4}, t_{5}, t_{6}, t_{7}$ and $t_{8}$. The duration between two adjacent decision epochs is 6 minutes. Two trajectory scenarios, as shown in Table $\mathrm{V}$, are considered.

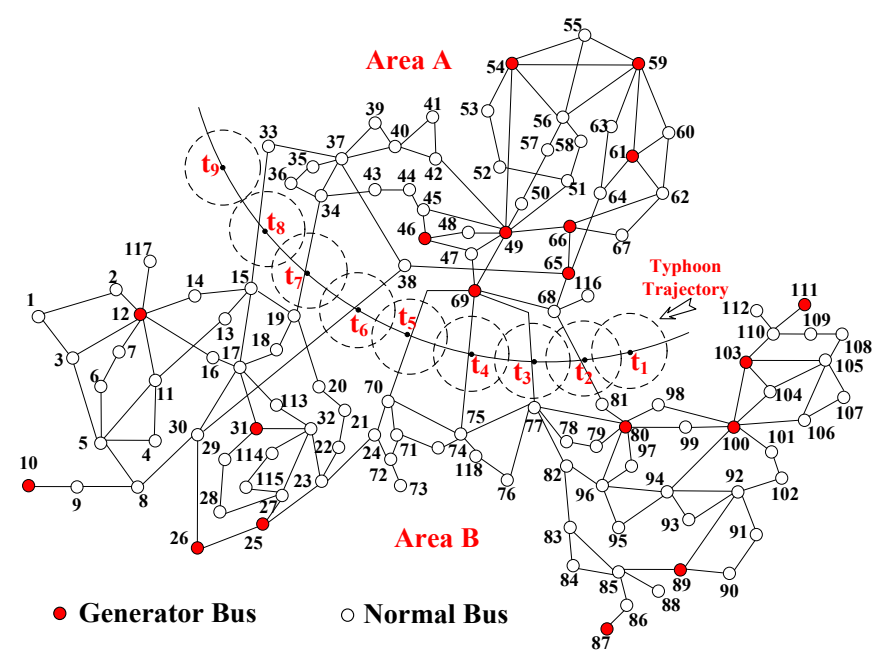

Fig. 11 IEEE 118-bus system topology and the typhoon trajectory.

\section{2) Simulation results of scenario one}

This section shows the influences on power flows. The trajectory of the typhoon divides the system into two areas A and B. In the first period, the area A has 1,670 MW load and $1,812 \mathrm{MW}$ real output, and the area B has 2,572 MW load and 2,430 MW real output. Since the DC power flow is used, line losses are ignored. The imbalance of real power outputs between $\mathrm{A}$ and $\mathrm{B}$ indicates that real power is transferred from $\mathrm{A}$ 
to $\mathrm{B}$ through the lines on the trajectory. When performing generation redispatch based on system states, the real output in the area A decreases and the real output in the area B increases, as shown in Fig. 12, to reduce real power through transmission lines on the trajectory of the typhoon, in case these line might be in failure.

TABLE V

DIFFERENT MAXIMUM FAILURE COMPONENT SCENARIOS

\begin{tabular}{|c|c|c|c|c|}
\hline \multirow{2}{*}{$\begin{array}{c}\text { Time } \\
\text { Period }\end{array}$} & \multicolumn{2}{|c|}{ Scenario one } & \multicolumn{2}{|c|}{ Scenario two } \\
\hline & Component & $\begin{array}{c}\text { Failure } \\
\text { Probability }\end{array}$ & Component & $\begin{array}{c}\text { Failure } \\
\text { Probability }\end{array}$ \\
\hline \multirow{2}{*}{$t_{2}$} & \multirow{2}{*}{ Line 68-81 } & \multirow{2}{*}{0.25} & Line 68-81 & 0.25 \\
\hline & & & $60 \%$ Load at bus 116 & 0.10 \\
\hline$t_{3}$ & Line 69-77 & 0.22 & Line 69-77 & 0.22 \\
\hline \multirow{2}{*}{$t_{4}$} & \multirow{2}{*}{ Line 69-75 } & \multirow{2}{*}{0.20} & Line 69-75 & 0.20 \\
\hline & & & Generator at bus 69 & 0.15 \\
\hline \multirow{2}{*}{$t_{5}$} & \multirow{2}{*}{ Line 69-70 } & \multirow{2}{*}{0.18} & Line 69-70 & 0.18 \\
\hline & & & $60 \%$ load at bus 70 & 0.10 \\
\hline$t_{6}$ & Line $29-38$ & 0.15 & Line $29-38$ & 0.15 \\
\hline \multirow{2}{*}{$t_{7}$} & \multirow{2}{*}{ Line 19-34 } & \multirow{2}{*}{0.12} & Line 19-34 & 0.12 \\
\hline & & & $50 \%$ load at bus 34 & 0.10 \\
\hline$t_{8}$ & Line $15-33$ & 0.10 & Line $15-33$ & 0.10 \\
\hline
\end{tabular}

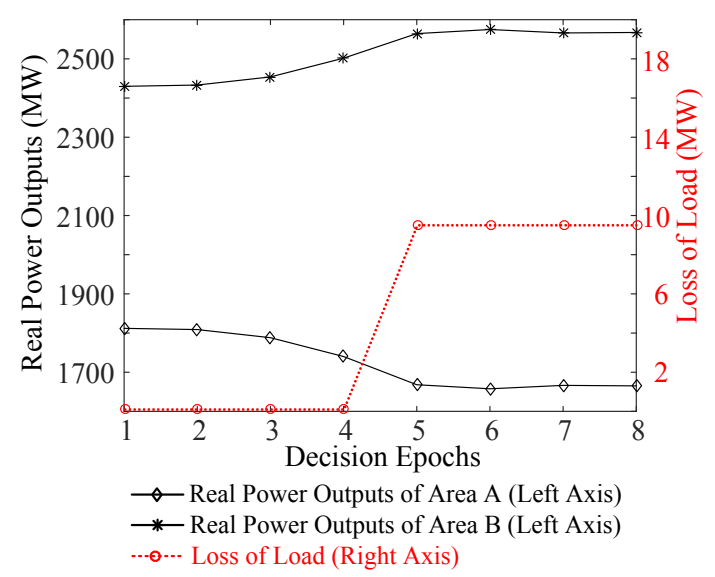

Fig. 12 Real power outputs and loss of load under the scenario that the lines $69-81,69-77,69-75,69-70$ and 30-38 are sequentially in failure at $t_{2}, t_{3}, t_{4}, t_{5}$ and $t_{6}$, respectively.

\section{3) Simulation results of scenario two}

This section shows the influences on outputs of generators on the trajectory of the typhoon. Since the generator at bus 69 may be in failure at the $4^{\text {th }}$ decision epoch with the probability 0.15 , its outputs remain close to its lower limit from the $1^{\text {st }}$ to $3^{\text {rd }}$ decision epochs, as shown in Fig. 13. This can avoid a large loss of load due to power unbalance considering operation constraints, e.g., ramping rates and upper limits of line capacity. If the generator is out of service at the $4^{\text {th }}$ decision epoch, its outputs at the $4^{\text {th }}$ and following decision epochs are zeros.

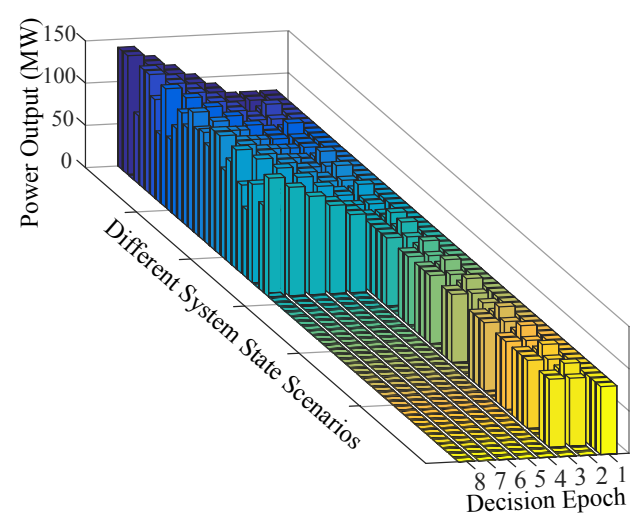

Fig. 13 Sequential outputs of the generator at bus 69 under different system state scenarios

\section{The Simplified Guangdong Power Grid System}

The simplified Guangdong power grid has 116 generating units, 1,117 buses and 1,771 transmission lines. We take the typhoon Mujigae [40], which struck the Guangdong province of China on October 4, 2015, as an example. According to the trajectory, seven critical transmission lines and one critical generator undergo the typhoon sequentially. The duration between two adjacent decision epochs is about one hour. The failure probabilities of the seven transmission lines and the generator on the trajectory are $0.2,0.18,0.12,0.1,0.080 .08$, 0.07 and 0.1 , respectively. The upper and lower limits of the critical generator are $1059 \mathrm{MW}$ and $110 \mathrm{MW}$. Its ramping rate is $100 \mathrm{MW} / \mathrm{h}$.

The benchmark strategy is based on the M2 method, which was introduced previously. Fig. 14 shows the differences of loss of load with M1 and M2. The results show that the proposed strategy (M1) can ensure a better performance compared with non-proactive strategies (M2).

Fig. 15 shows the outputs of the critical generator on the trajectory. Since the critical generator may be in failure at the $5^{\text {th }}$ decision epoch, its outputs remain close to its lower limit from the $1^{\text {st }}$ to $4^{\text {th }}$ decision epochs to avoid a large loss of load due to power unbalance considering operation constraints.

Fig. 16 shows the cost of expected loss of load with different numbers of available generators for dispatch. Each value is the average value based on 50 simulations, which randomly select generators for dispatch. Results show that more generators for dispatch tend to be less costs of expected loss of load.

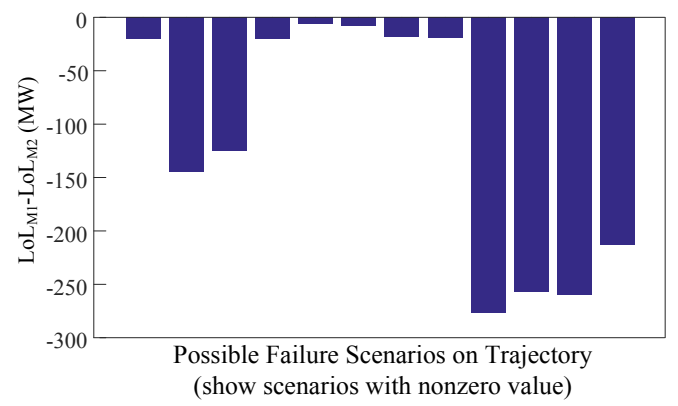

Fig. 14 Differences of LoL with M1 and M2. 


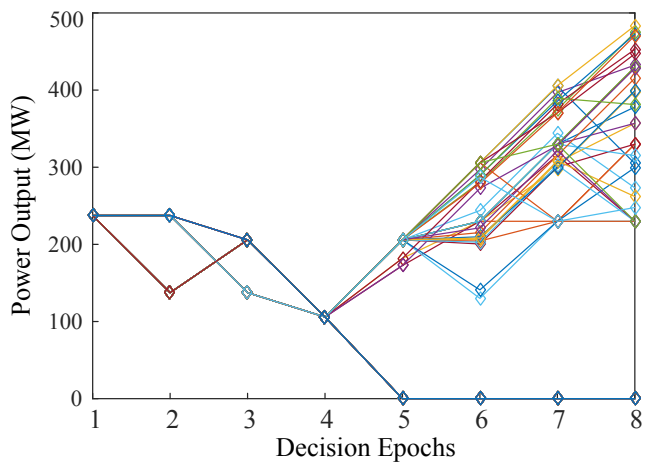

Fig. 15 Outputs of the critical generator on the trajectory

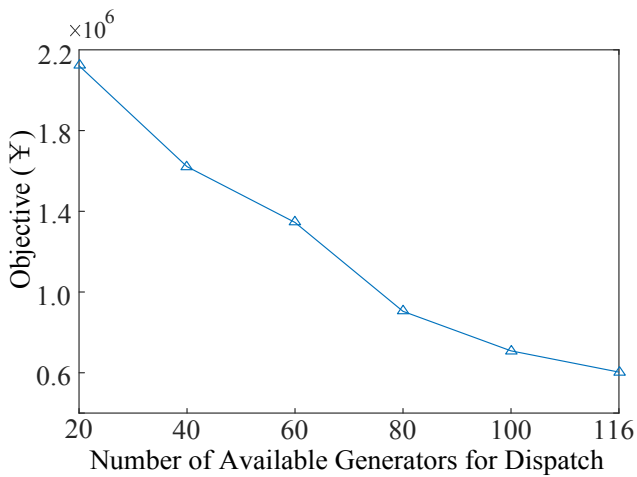

Fig. 16 Objective values with different numbers of available generators for dispatch

\section{CONCLUSIONS}

In this paper, a Markov model was proposed to construct sequentially proactive generation redispatch strategies based on system states, in consideration of operating constraints. Since trajectories and the failures of components on the trajectory due to extreme weather events are with uncertainties, a recursive value function that included the current cost and the future cost for each state was established. To address the computational difficulties caused by the constraints of ramping rates of generators, the recursive value function was transformed into a mixed integer linear programming model, with the probability of each state used as the weight of each objective. Simulations were carried out on the IEEE 30-bus system, the IEEE 118-bus system and a simplified Guangdong power grid system to validate the proposed model. The major findings are as follows. 1) The Markov model can adequately describe sequential decision processes with consideration of uncertainties during an event unfolding. 2) The proposed strategies tend to redispatch the power flows of lines on the trajectory of the extreme weather event to reduce potential power loss. 3) The proposed model provides insight into proactive operation strategies under extreme weather events. The results demonstrate that the proposed proactive operation strategies can reduce loss of load due to the development of extreme events.

\section{ACKNOWLEDGE}

The authors would like to thank Prof. Chen-Ching Liu with
Washington State University for insightful suggestions and helpful discussions.

\section{REFERENCES}

[1] Department of Energy(DoE). Insurance as a Risk Management Instrument for Energy Infrastructure Security and Resilience, Mar. 2013.[Online]. Available: http://energy.gov/oe/downloads/insurancerisk-management-instrument-energy-infrastructure-security-and-resilien $\underline{\text { ce }}$

[2] International Energy Agency (IEA). RD\&D Needs for Energy System Climate Preparedness and Resilience, Nov. 2013.[Online]. Available: https://www.iea.org/media/workshops/2013/egrdutrecht/WorkshopSum maryNov2013.pdf

[3] National Academy of Sciences U.S.A, National Research Council: Disaster Resilience: A National Imperative. Washington, D.C.: The National Academies Press, 2012.

[4] Executive Office of the President. U.S.A., Economic Benefits of Increasing Electric Grid Resilience to Weather Outages, Aug. 2013. [Online]. Available: http://energy.gov/downloads/economic-benefitsincreasing-electric-grid-resilience-weather-outages

[5] Executive Office of the President. U.S.A., A policy framework for the $21^{\text {st }}$ century grid, 2011. [Online]. Available: https://www.whitehouse.gov/sites/default/files/microsites/ostp/nstc-smar t-grid-june2011.pdf

[6] House of Lords. U.K., Science and Technology Select Committee, The Resilience of the Electricity System, Mar. 2015.[Online]. Available: http://www.publications.parliament.uk/pa/ld201415/ldselect/ldsctech/12 1/121.pdf

[7] North American Electric Reliability Corporation (NERC). High-Impact, Low-Frequency Event Risk to the North American Bulk Power System, Jun. 2010. [Online]. Available: http://www.nerc.com/pa/CI/Resources /Documents/HILF\%20Report.pdf

[8] North American Electric Reliability Corporation (NERC). Severe Impact Resilience: Considerations and Recommendations, May 2012. [Online]. Available: http://www.nerc.com/docs/oc/sirtf/SIRTF_Final_May_9 2012-Board_Accepted.pdf

[9] North American Electric Reliability Corporation (NERC). Electricity Sub-sector Coordinating Council: Critical Infrastructure Strategic Roadmap, Nov. 2010. [Online]. Available: http://www.nerc.com/pa/CI/ Pages/ESCC.aspx

[10] Electric Power Research Institute (EPRI). U.S.A., Enhancing Distribution Resiliency: Opportunities for Applying Innovative Technologies, Jan. 2013.[Online]. Available: http://www.epri.com/abstracts/Pages/ ProductAbstract.aspx?ProductId $=000000000001026889$

[11] M. Panteli and P. Mancarella, "The grid: stronger, bigger, smarter?: presenting a conceptual framework of power system resilience," IEEE Power Energy Mag., IEEE, pp. 58-66, 2015.

[12] H. Liu, R. A. Davidson, J. R. Stedinger, D. V. Rosowsky, and D. V. Rosowsky, "Negative binomial regression of electric power outages in hurricanes," Journal of Infrastructure Systems, vol. 11, no. 4, pp. 258-267, 2005

[13] S. D. Guikema, R. Nateghi, S. M. Quiring, A. Staid, A. C. Reilly, and M. Gao, "Predicting hurricane power outages to support storm response planning," IEEE Access, vol. 2, pp. 1364-1373, 2014.

[14] R. Nateghi, S. Guikema, and S. Quiring, "Forecasting hurricane-induced power outage durations," Natural Hazards, vol. 74, no. 3, pp. 1795-1811, 2014.

[15] M. Panteli and P. Mancarella, "Modeling and evaluating the resilience of critical electrical power infrastructure to extreme weather events," Systems Journal, IEEE, DOI: 10.1109/JSYST.2015.2389272, early access article.

[16] A. Arab, A. Khodaei, S. K. Khator, K. Ding, V. A. Emesih, and Z. Han, "Stochastic pre-hurricane restoration planning for electric power systems infrastructure," IEEE Trans. Smart Grid, vol. 6, pp. 1046-1054, 2015.

[17] W. Sun, C. C. Liu, and S. Liu, "Black start capability assessment in power system restoration," IEEE Power and Energy Society General Meeting, San Diego, CA, 2011, pp. 1-7.

[18] F. Qiu, J. Wang, C. Chen, and J. Tong, "Optimal black start resource allocation," IEEE Trans. Power Syst., vol. 31, no. 3, pp. 2493-2494, 2016.

[19] J. Shang, X. Sheng, J. H. Zhang, and W. Zhao, "The optimized allocation of mobile emergency generator based on the loads importance," Asia-Pacific Power and Energy Engineering Conference, Wuhan, China, 2009, pp. 1-4. 
[20] C. Wang, Y. Hou, Z. Qin, C. Peng, and H. Zhou. "Dynamic coordinated condition-based maintenance for multiple components with external conditions," IEEE Trans. Power Del., vol. 30, no. 5, pp. 2362-2370, 2015.

[21] S. Zhang and V. Vittal, "Wide-area control resiliency using redundant communication paths," IEEE Trans. Power Syst., vol. 29, pp. 2189-2199, 2014.

[22] S. Zhang and V. Vittal, "Design of wide-area power system damping controllers resilient to communication failures," IEEE Trans. Power Syst., vol. 28, pp. 4292-4300, 2013.

[23] W. Yuan, J. Wang, F. Qiu, C. Chen, C. Kang, and B. Zeng, "Robust optimization-based resilient distribution network planning against natural disasters," IEEE Trans. Smart Grid, DOI: 10.1109/TSG.2015.2513048, early access article.

[24] M. Panteli, D. N. Trakas, P. Mancarella, and N. D. Hatziargyriou, "Boosting the power grid resilience to extreme weather events using defensive islanding," IEEE Trans. Smart Grid, DOI: 10.1109/ TSG.2016.2535228, early access article.

[25] Z. Wang and J. Wang, "Self-Healing resilient distribution systems based on sectionalization into microgrids," IEEE Trans. Power Syst., vol. 30, no. 6, pp. 3139-3149, 2015.

[26] M. M. Adibi and L. H. Fink, "Overcoming restoration challenges associated with major power system disturbances," IEEE Power Energy Mag., vol. 4, no. 5, pp. 68-77, 2006.

[27] Y. Hou, C.-C. Liu, K. Sun, P. Zhang, S. Liu, and D. Mizumura, "Computation of milestones for decision support during system restoration," IEEE Trans. Power Syst., vol. 26, no.3, pp.1399-1409, 2011.

[28] C. Chen, J. Wang, F. Qiu, and D. Zhao, "Resilient distribution system by microgrids formation after natural disasters" IEEE Trans. Smart Grid, vol. 7, no. 2, pp. 958 - 966, 2016.

[29] L. H. Fink, K.-L. Liou and C.-C. Liu, "From generic restoration actions to specific restoration strategies," IEEE Trans. Power Syst., vol. 10, no. 2, pp. 745-752, 1995.

[30] W. Sun, C. C. Liu and L. Zhang, "Optimal generator start-up strategy for bulk power system restoration," IEEE Trans. Power Syst., vol. 26, no. 3, pp. 1357-1366, 2011.

[31] C. C. Liu, S. J. Lee and S. S. Venkata, "An expert system operational aid for restoration and loss reduction of distribution systems," IEEE Trans. Power Syst., vol. 3, no. 2, pp. 619-626, 1988.

[32] S. Toune, H. Fudo, T. Genji, Y. Fukuyama and Y. Nakanishi, "Comparative study of modern heuristic algorithms to service restoration in distribution systems," IEEE Trans. Power Del., vol. 17, no. 1, pp. 173-181, Jan 2002.

[33] A. Castillo, "Microgrid provision of blackstart in disaster recovery for power system restoration," Smart Grid Communications, IEEE International Conference on, Vancouver, BC, 2013, pp. 534-539.

[34] F. Ren, M. Zhang, D. Soetanto and X. Su, "Conceptual design of a multi-agent system for interconnected power systems restoration," IEEE Trans. Power Syst., vol. 27, no. 2, pp. 732-740, 2012.

[35] Y. Wang, C. Chen, J. Wang and R. Baldick, "Research on resilience of power systems under natural disasters - a review," IEEE Trans. Power Syst., vol. 31, no. 2, pp. 1604-1613, 2016.

[36] G. Li, P. Zhang, P. B. Luh, W. Li, Z. Bie, C. Serna, et al., "Risk analysis for distribution systems in the northeast U.S. under wind storms," IEEE Trans. Power Syst., vol. 29, no. 2, pp. 889-898, 2014.

[37] M. Puterman, Markov Decision Process. New York, USA: Wiley, 1994.

[38] IEEE 118-bus system data. [Online]. Available: https://www.ee.washington.edu/research/pstca/pf118/pg tcal18bus.htm

[39] IEEE 118-bus system data. [Online]. Available:http://motor.ece.iit.edu/ DC/VSC/IEEE118.xls

[40] Trajectory of typhoon Mujigae. [Online]. http://www.hko.gov.hk/wxinfo/currwx/tc_pos_1519.htm

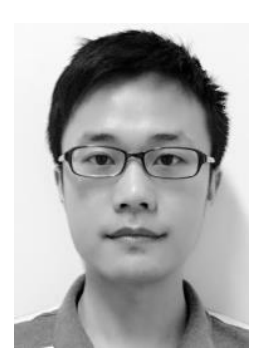

Chong Wang (S'15) received the B.E and M.S degrees in electrical engineering from Hohai University, Nanjing, China, in 2009 and 2012, and the $\mathrm{Ph} . \mathrm{D}$. degree in electrical engineering from The University of Hong Kong, Hong Kong, in 2016. Currently, he is a postdoctoral researcher with The University of Hong Kong.

His research interests include optimization and its applications in power systems, smart grids, integration of renewable energy sources, and cyber-physical systems.

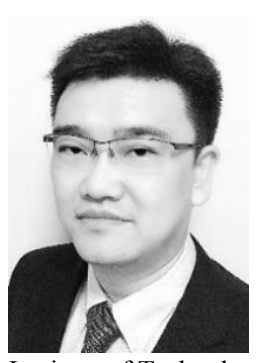

Yunhe Hou (M'08-SM'15) received the B.E. and $\mathrm{Ph} . \mathrm{D}$. degrees in electrical engineering from the Huazhong University of Science and Technology, Wuhan, China, in 1999 and 2005, respectively.

He was a Post-Doctoral Research Fellow at Tsinghua University, Beijing, China, from 2005 to 2007, and a Post-Doctoral Researcher at Iowa State University, Ames, IA, USA, and the University College Dublin, Dublin, Ireland, from 2008 to 2009. He was also a Visiting Scientist at the Laboratory for Information and Decision Systems, Massachusetts Institute of Technology, Cambridge, MA, USA, in 2010. He joined the faculty of the University of Hong Kong, Hong Kong, in 2009, where he is currently an Assistant Professor with the Department of Electrical and Electronic Engineering.

Dr. Hou is an Editor of the IEEE TRANSACTIONS ON SMART GRID and an Associate Editor of JOURNAL OF MODERN POWER SYSTEMS AND CLEAN ENERGY.

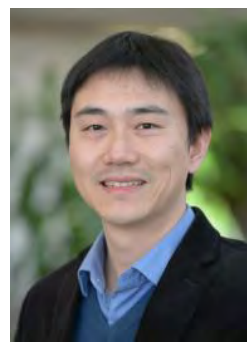

Feng Qiu (M'14) received his Ph.D. from the School of Industrial and Systems Engineering at the Georgia Institute of Technology in 2013. He is a computational scientist with the Energy Systems Division at Argonne National Laboratory, Argonne, IL, USA.

His current research interests include optimization in power system operations, electricity markets, and power grid resilience.

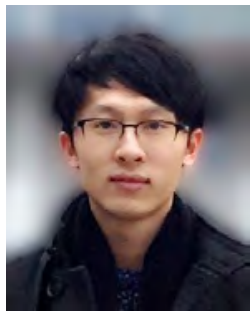

Shunbo Lei (S'14) received the B.E. degree from Huazhong University of Science and Technology, Wuhan, China, in 2013. He is currently pursuing the Ph.D. degree with The University of Hong Kong, Hong Kong. He is currently visiting Argonne National Laboratory, Argonne, IL, USA.

His research interests include optimization of power system operation, renewables integration, and power system resilience.

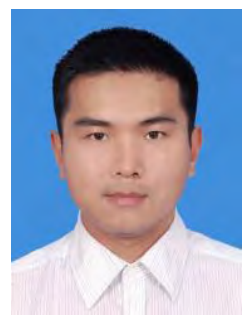

Kai Liu received the B.Sc. degree from Tsinghua University, Beijing, China, in 2005, and the M.Phil. and $\mathrm{Ph}$.D. degrees from the University of Hong Kong, Hong Kong, in 2007 and 2011, respectively, both in electrical engineering.

He currently works as a senior engineer in the dispatch and control center of the China Southern Power Grid (CSG). His research interests include the operations, economics, and planning of power systems. 